\title{
Investigation of the Effects of the Arc Fault Resistance and Transient Resistance on the Overcurrent Ground Relay Protection Setting in Medium Voltage Electrical Power Lines
}

\author{
Plamen Stanchev ${ }^{1}$, Mediha Mehmed-Hamza ${ }^{2}$ \\ 1 - Technical University of Varna, Department of Electric Power Engineering, 9010, 1 Studentska Street, Varna, Bulgaria \\ 2 - Medical University of Varna, Department of Medical Equipment, Electronic and Information Technologies in Health care, 9002 Varna, \\ Bulgaria
}

Corresponding author contact: p.stanchevetu-varna.bg

\begin{abstract}
In the medium voltage electrical power lines, the most common fault is the phase to ground fault. The determining factors for selection of the neutral grounding mode of the medium voltage electrical power lines are the selectivity of the relay protection, the transient voltages during phase to ground fault, the magnitude of the fault currents, etc. The paper studies the influence of the transient resistance of phase to ground fault on the selectivity and sensitivity of the earth fault protection when the neutral of the power transformer is grounded through active resistance in medium voltage power grids.
\end{abstract}

Keywords: medium voltage, phase to ground faults, earth fault relay protection

\section{$1 \quad$ Introduction}

The medium voltage power lines (MV) are the most common in Bulgaria with about $70 \%$ of their faults being phase to ground faults. xpansion/Expanding the medium voltage grid will also result in increased length and number of the cable lines. The most commonly used methods for grounding of the MV transformer neutral in Bulgaria are through active resistance and combined grounding - reactor in parallel to active resistance.

The operation of the electrical lines under normal conditions does not depend on the grounding of the neutral. For phase to ground faults, the grounding mode greatly defines the requirements for the insulation level of the line and the equipment that is electrically connected to it. The grounding mode also affects the level of the switching overvoltages and the methods for their limitation, the magnitude of the currents in the earth fault point, the operation of the relay protection, the safety conditions, the capital investments for the construction of the line, etc. (Shabad, 2002; Shabad, 2004; Etarski, 1994, Yordanova, 2016).

Grounding modes and their pros and cons are well known. The determining factors for selecting the neutral grounding mode are the type of power grid and the magnitude of the phase-to-ground fault current. Regulatory requirements for cable lines require grounding of the neutral via active resistance. For mixed overhead and cable lines - through active resistance when the cable part share is greater than $40 \%$ of the total grid length. And combined (reactor with active resistance) when the overhead line share is more than 60\% (Ministry of Energy Ordinance № 3, 2015; Ministry of Energy Ordinance № 16-116, 2015).

The purpose of the paper is to investigate the influence of the transient resistance of the phase to ground fault on the selectivity and sensitivity of the earth fault protection when the MV transformer neutral is grounded through active resistance.

\section{Model scheme and settings of the earth fault relay protection}

Considered hereto is a medium voltage electrical line with a total length of $130 \mathrm{~km}$, composed of overhead and cable lines,. The power transformer is rated at $40 \mathrm{MVA}$ and the neutral is grounded through a $40 \Omega$ resistor. The investigated electrical line has $40 \%$ of underground cables and the remaining 60 
$\%$ are overhead sections. The studies on the change of the electrical variables and the effect of the arc resistance at ground fault point were conducted in MATLAB Simulink software environment.

The model of the studied lines is shown in Fig. 1.

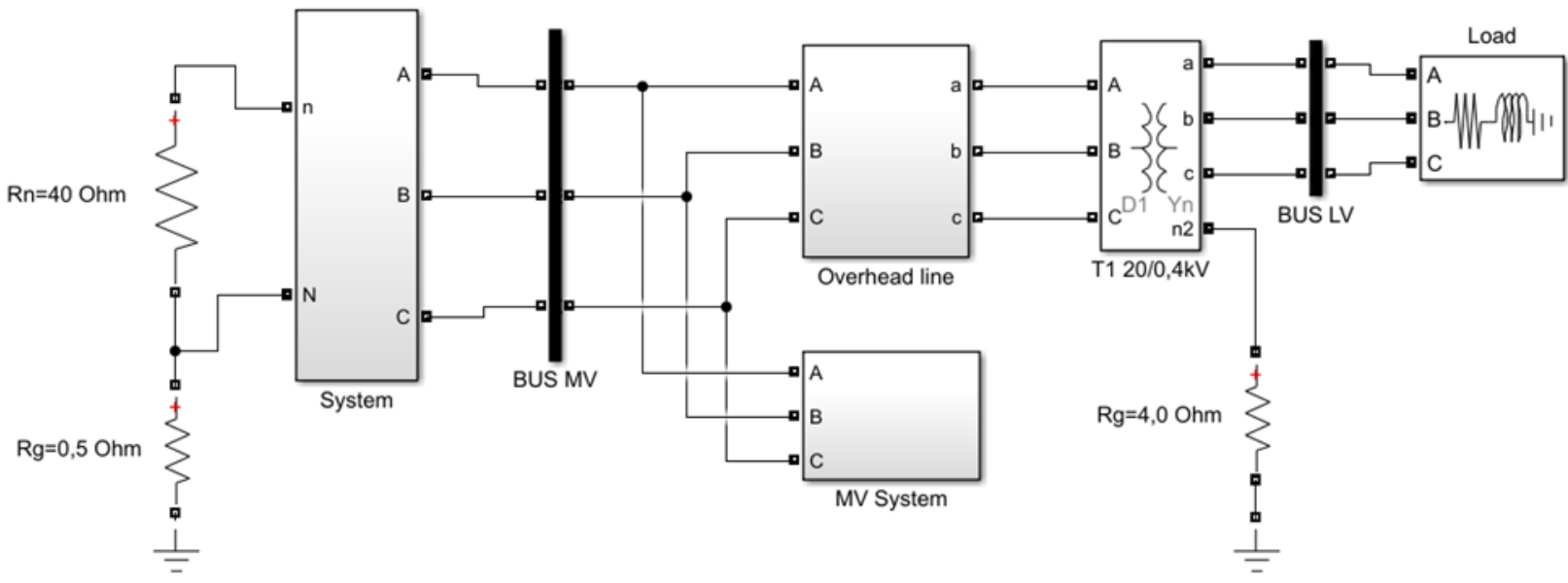

Fig. 1. Model scheme for the studied line

Earth fault protection can be used to protect against phase to earth faults in MV electrical power lines with a grounded neutral through active resistance. The setting of earth relay protection covers the calculation of the thershold current, the time delay and the sensitivity of the relay protection. In order to increase the selectivity and sensitivity of earth fault relay protection, a three-stage earth protection is preferably used, which takes into account the effect of the transient resistance in earth damage.

The primary threshold current $I_{R P}$ of the first stage of earth protection determined by (Avramov, 1986; Andreev, 2005; Vichev \& Todorova, 1998; Domeland, 1981; Fedoseev, 1976; TSO LTD).

$$
I_{R P}=K_{\text {security }} \cdot K_{\text {discharge }} . I_{C 0}, \mathrm{~A}
$$

where: $K_{\text {security }}=1,1 \div 1,2$ is the security coefficient, $K_{\text {discharge }}$ is the coefficient allowing for the sudden discharge of the cable line in the case of external faults and equals to: $4 \div 5$ - in the absence of delay in relay protection, $2 \div 3$ - in the presence of delay in the relay protection, 1 - when the relay protection acts on a signal, $I_{C 0}$ is the zero-sequence overhead line capacity.

The first stage is instantaneous (without delay) and the start time of the first stage is $t=0 \mathrm{~s}$.

The second stage of the earth relay protection recognizes the impact of transient resistance at the location of the fault. In order to provide sufficient sensitivity of the earth fault protection at ground connection through high transient resistance, the threshold current of the second stage is determined by the earth fault current with an arc resistance $100 \Omega$ (TSO LTD).

$$
I_{R P}=\frac{I_{L-G 100 \Omega}}{K_{\text {sensitivity }}}, \mathrm{A}
$$

where $I_{L-G 100 \Omega}$ is the earth fault current at failure through a transient impedance of $100 \Omega$.

The second stage of the earth fault protection is without delay $t=0 \mathrm{~s}$.

The sensitivity coefficient $K_{\text {sensitivity }}$ is used to check the sensitivity of the protection in line with (Ministry of Energy Ordinance № 3, 2015; Avramov, 1986; Andreev, 2005; Vichev \& Todorova, 1998; Domeland, 1981; Fedoseev, 1976; TSO LTD):

$$
K_{\text {sensitivity }}=\frac{I_{L-G}}{I_{R P}}, \mathrm{~A}
$$

where $I_{L-G}$ is the estimated current at zero arc resistance phase to ground fault at the end of the line. 
For cable lines $K_{\text {sensitivity }} \geq 1,25$, for overhead lines - 1,5 for the first stage and 2,5 for the second stage.

The current threshold setting of the third stage provides sensitivity of the protection for phase to ground fault through high arc resistance. It is performed with the current threshold set at $10 \mathrm{~A}$. Subsequently, the time delay is $t=3,6 \mathrm{~s}$ and acts on the signal (TSO LTD).

\section{$3 \quad$ Results and analysis}

Investigated, for the purposes of the surveyed MV line, was the variation of the electrical variables for earth faults through an arc resistance of $0.001 \Omega$ to $100 \Omega$. The examined variation of the magnitude of the phase to ground fault current for the overhead power lines constructed with ACSR 70 and ACSR 95 conductors revealed that their length varied from 10 to $40 \mathrm{~km}$.

Presented in Table 1 are the results for the value of the phase to ground fault current at an arc resistance from $0.001 \Omega$ to $100 \Omega$ for an ACSR 70 conductors.

Table 1. Phase to Grund fault current at an arc resistance of $0.001 \Omega$ to $100 \Omega$ for an ACSR 70 conductor

\begin{tabular}{|c|c|c|c|c|}
\hline Rarc, $\mathbf{\Omega}$ & $\begin{array}{c}\text { ACSR 70 } \\
\mathbf{1 0 k m}\end{array}$ & $\begin{array}{c}\text { ACSR 70 } \\
\mathbf{2 0 k m}\end{array}$ & $\begin{array}{c}\text { ACSR 70 } \\
\mathbf{3 0 k m}\end{array}$ & $\begin{array}{c}\text { ACSR 70 } \\
\mathbf{4 0 k m}\end{array}$ \\
\hline 0,001 & 274 & 261 & 241 & 219 \\
\hline 20 & 192 & 183 & 173 & 161 \\
\hline 40 & 147 & 141 & 135 & 128 \\
\hline 60 & 119 & 115 & 110 & 105 \\
\hline 80 & 100 & 97 & 93 & 90 \\
\hline 100 & 86 & 83 & 81 & 78 \\
\hline
\end{tabular}

With increasing the In short, increased arc resistance of the fault produces decreased value of the phase to ground fault current.

Figure 2 illustrates the results obtained for the effect of the arc resistance on the value of the phase to ground fault current for conductor ACSR 70 overhead line with $10 \mathrm{~km}$ and $40 \mathrm{~km}$ lengths for the tested electrical line.

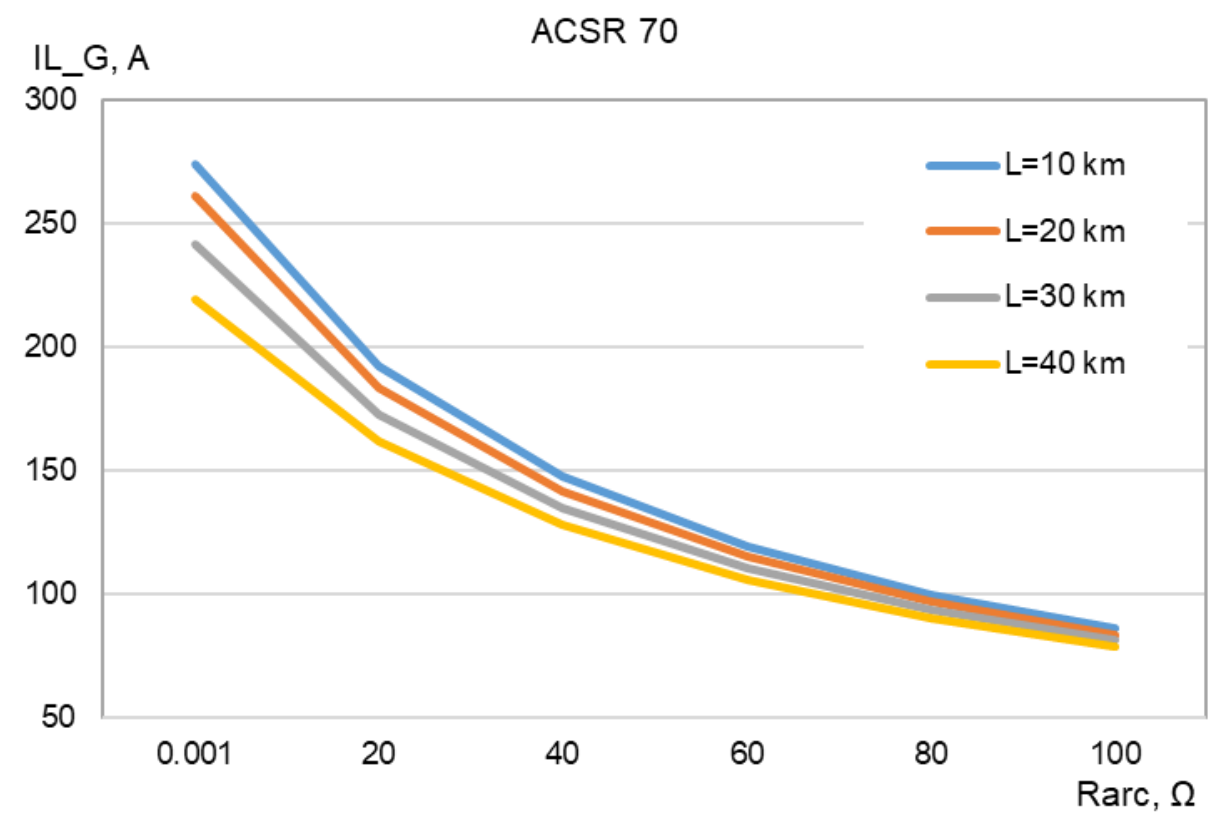

Fig. 2. Effect of the transient impedance on the value of the phase to ground fault current

Figure 3 shows the results obtained for the value of the phase to ground fault current at an arc resistance of $100 \Omega$. 


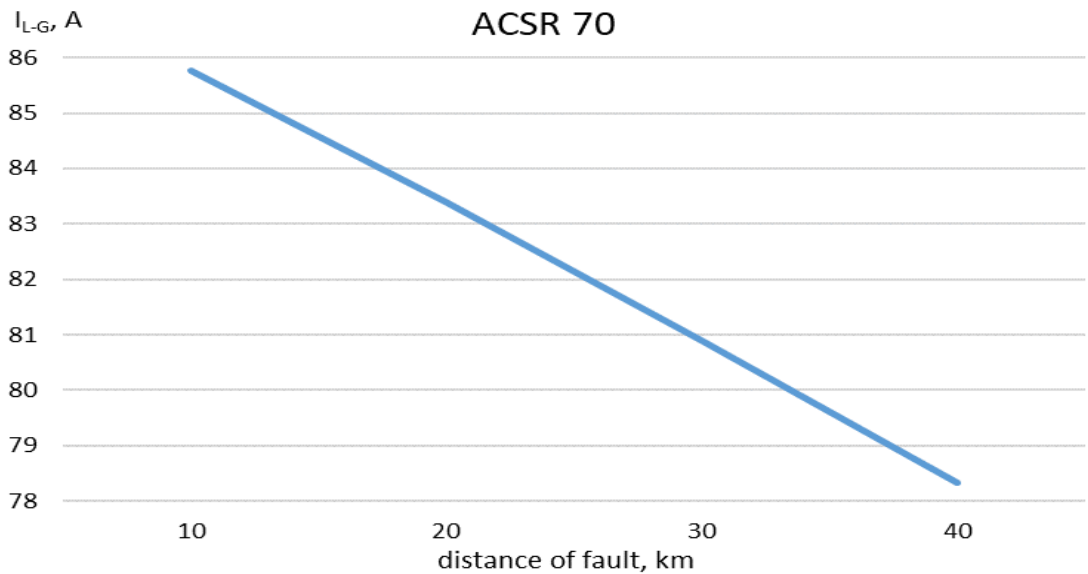

Fig. 3. Values of the phase to ground fault current at a transient resistance of $100 \Omega$

Displayed in Table 2 are the results for the value of the phase-to-ground fault current at a transient resistance of $0.001 \Omega$ to $100 \Omega$ for an ACSR 95 conductor.

Table 2. The earth fault current at a transient resistance of $0.001 \Omega$ to $100 \Omega$ for an ACSR 95 conductor

\begin{tabular}{|c|c|c|c|c|}
\hline Rarc, $\mathbf{\Omega}$ & $\begin{array}{c}\text { ACSR 95 } \\
\mathbf{1 0 k m}\end{array}$ & $\begin{array}{c}\text { ACSR 95 } \\
\mathbf{2 0 k m}\end{array}$ & $\begin{array}{c}\text { ACSR 95 } \\
\mathbf{3 0 k m}\end{array}$ & $\begin{array}{c}\text { ACSR 95 } \\
\mathbf{4 0 k m}\end{array}$ \\
\hline 0,001 & 279 & 271 & 255 & 235 \\
\hline 20 & 195 & 188 & 180 & 170 \\
\hline 40 & 149 & 144 & 139 & 133 \\
\hline 60 & 120 & 117 & 113 & 109 \\
\hline 80 & 100 & 98 & 95 & 92 \\
\hline 100 & 86 & 84 & 82 & 80 \\
\hline
\end{tabular}

Figure 4 indicates the results obtained for the effect of the transient impedance on the value of the phase to ground fault current for conductor ACSR 95 overhead line with $10 \mathrm{~km}$ and $40 \mathrm{~km}$ lengths for the tested electrical line.

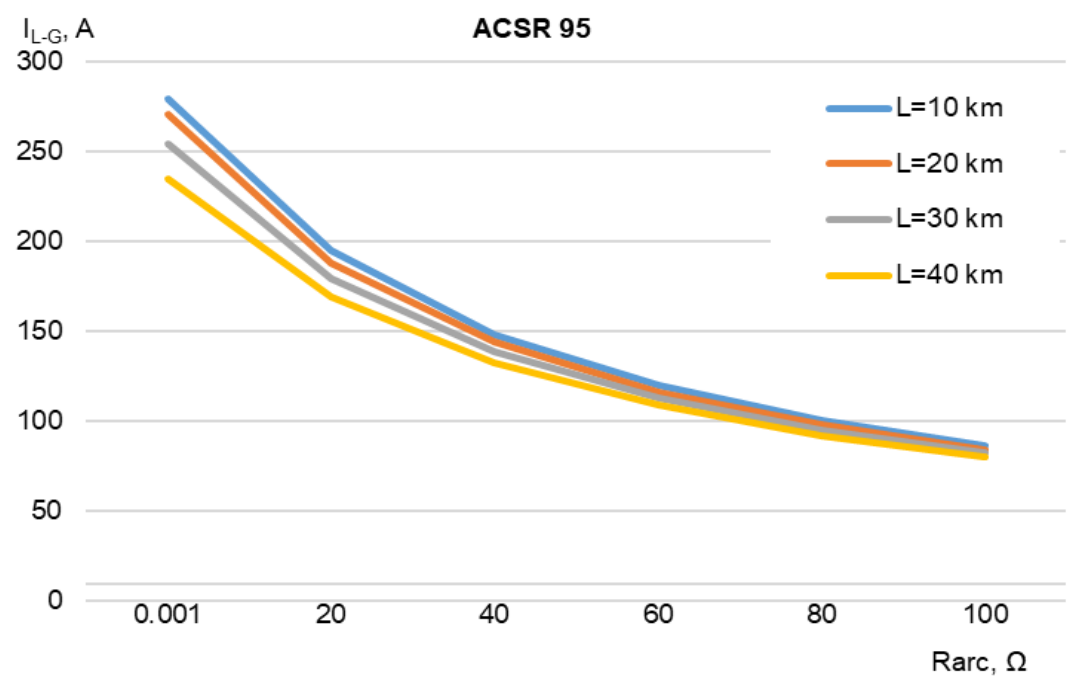

Fig. 4. Effect of the transient impedance on the value of the phase to ground fault current

Figure 5 shows the results obtained for the value of the phase to ground fault current at a transient resistance of $100 \Omega$. 


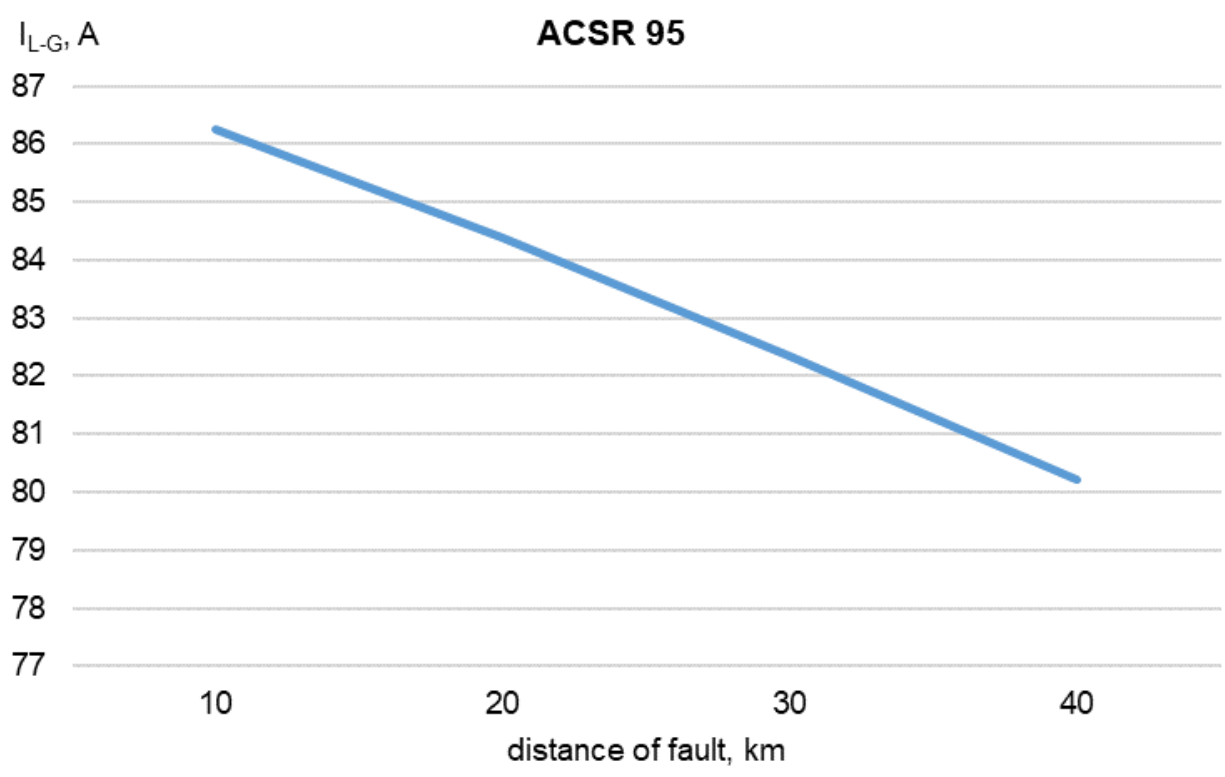

Fig. 5. Values of the phase to ground fault current at a transient resistance of $100 \Omega$

To determine the sensitivity factor of the first stage of the earth fault protection, it is necessary to know the magnitude of the fault current for a zero arc resistance phase-to-ground fault at the end of the line. The results derived for the investigated lines can be further used to determine the coefficient of sensitivity.

In the setting of the second stage of earth fault protection, applied is the magnitude of the current for a phase-to-ground fault through a $100 \Omega$ arc fault.

For a phase-to-ground fault through a transient resistance, the value of the fault current is reduced, which leads to decreased sensitivity of the protection. For a phase to ground fault through a transient impedance of $100 \Omega$ at the end of a $40 \mathrm{~km}$ long terminal, the earth connection current decreases by about $65 \%$

Taking $K_{\text {security }}=1,1$ and $K_{\text {discharge }}=5$ after substitution in (1) and (3) translates into:

$$
I_{C 0} \leq \frac{I_{L-G}}{8,25}
$$

Formula 4 gives the restrictive condition for setting the first stage of the earth fault relay protection. In order to meet the prerequisite for a first-rate sensitivity coefficient for the selected values of the coefficients, it is necessary to fulfill the requirement of formula 4.

\section{Conclusion}

1. Results have been obtained for the range of variation of the phase-to-ground fault current depending on the value of the arc fault resistance. The results achieved can be used to adjust the sensitivity of the first stage of earth protection.

2. The outcomes produced for changes in the value of the phase to ground fault current at a transient impedance of $100 \Omega$, can be further used to set the second stage of earth fault protection.

3. A formula has been devised for the threshold value of the zero-sequence capacitive current at which the condition for the sensitivity of the first stage of earth fault relay protection is fulfilled.

\section{References}

Shabad, M. A. (2002). Protection against single-phase short circuits to earth in 6-35 kV networks. Sank Petersburg 
Shabad, M. A. (2004). Grounding modes have not neutralized and protection against earth faults in 6$35 \mathrm{kV}$ networks of Russia. International Scientific and Technical Conference, Issue 3, Minsk, $337-342$.

Etarski, S.I. (1994). Electrical part of power plants. Sofia, Technology.

Yordanova, M., Vasileva, M., Dimitrova, R., Ivanova, M. (2016), A methodology for determining the maximum touch voltage in the grounding grids considering the impulse resistance. 19th International Symposium on Electrical Apparatus and Technologies, SIELA 2016, 376-379. https://doi.org/10.1109/SIELA.2016.7543067

Ministry of Energy. (2015). Ordinance № 3 Regarding the construction of electrical switchyards, switchgears and power lines. Retrieved from http://www.gli.government.bg/page.php?c=34\&page $=8$ (in Bulgarian).

Ministry of Energy. (2015). Ordinance № 16-116 for the technical operation of energy equipment. Retrieved from http://www.gli.government.bg/page.php?c=34\&page=8 (in Bulgarian).

Avramov, N. A. (1986). Fundamentals of Relay Protection. Sofia, Technology.

Andreev, S. (2005). Relay Protection. Varna.

Vichev, S., Todorova, A. et al.(1998). Energy Directory. Sofia, ABS Engineering, vol. 4

Domeland, V. (1981). Handbook of Relay Protection, Engineering.

Fedoseev, A. M. (1976). Relay Protection of the Electric Power Systems. Moskow, Energy

TSO LTD. Bulgaria Instruction for organization of relay protections and calculation of their settings in the medium voltage distribution networks. in Bulgarian 